\title{
Spiders can be recognized by counting their legs
}

\author{
Sarah Berkemer, Ricardo Chaves, Adrian Fritz, Marc Hellmuth, Maribel \\ Hernandez-Rosales and Peter F. Stadler
}

To all arachnophobic mathematicians.

\begin{abstract}
Spiders are arthropods that can be distinguished from their closest relatives, the insects, by counting their legs. Spiders have 8 , insects just 6 . Spider graphs are a very restricted class of graphs that naturally appear in the context of cograph editing. The vertex set of a spider (or its complement) is naturally partitioned into a clique (the body), an independent set (the legs), and a rest (serving as the head). Here we show that spiders can be recognized directly from their degree sequences through the number of their legs (vertices with degree 1). Furthermore, we completely characterize the degree sequences of spiders.
\end{abstract}

Mathematics Subject Classification (2010). Primary 05C07; Secondary 05C75.

Keywords. Phylogenetics, Cograph, P4-sparse, Spider, Degree Sequence.

\section{Introduction}

Determining the evolutionary history of species based on sequence information is one of the main challenges in phylogenomics. Recent advances in phylogenetics have shown that cographs play a crucial role in order to clean up orthology, resp., paralogy data, i.e., estimates of pairs of genes that arose from a speciation, resp., duplication event in the gene tree [4, 5. However, the (decision version of the) problem to edit a given graph into a cograph is NP-complete 9, 10. Nevertheless, the cograph editing problem can be solved in polynomial time, whenever the graph under investigation is so-called $P_{4}$-sparse [9, 10]. The structure of $P_{4}$-sparse graphs can be characterized in terms of so-called spiders 7. 8. Hence, the cograph editing problem can be rephrased as a "spider editing problem". To address the spider editing problem in future work, we give in this contribution a full characterization of spiders in terms of their degrees and respective degree sequences.

\section{Preliminaries}

We consider only simple graphs $G=(V, E)$. The degree $\operatorname{deg}_{G}(v)$ of a vertex $v \in V$ is defined as the number of edges that contain $v$. If there is no risk of confusion we write $\operatorname{simply} \operatorname{deg}(v) \operatorname{instead}$ of $\operatorname{deg}_{G}(v)$. The degree sequence $\left(n_{0}, n_{1}, n_{2} \ldots, n_{w}\right)$ is a list of non-negative integers. A degree sequence is graphical, if it can be realized by a graph, i.e., if there is a graph $G$ s.t. there are exactly $n_{k}$ vertices with degree $k$ in $G$. Of course, for simple graphs we have $n_{k}=0$ for $k \geq|V|$. It is well-known that one can verify in polynomial time, whether a degree sequence is graphical, either by means of the Havel-Hakimi algorithm [3, 2] or by using the Erdős-Gallai Theorem [1].

A graph $H$ is a subgraph of a graph $G$, in symbols $H \subseteq G$, if $V(H) \subseteq V(G)$ and $E(H) \subseteq E(G)$. A subgraph $H \subseteq G$ is called induced, when $x y \in E(H)$ if and only if $x y \in E(G)$ for all $x, y \in$

This work was funded in part by the German Research Foundation (DFG) (Proj. No. MI439/14-1). 


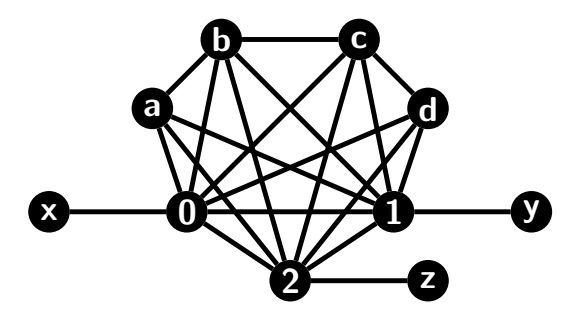

Figure 1. This thin spider, with vertex partition $K=\{0,1,2\}, S=\{x, y, z\}$ and $R=\{a, b, c, d\}$ has degree sequence $(0,3,0,0,2,2,0,3)$. In this example there is another spider hidden in the head $R$, namely $K^{\prime}=\{b, c\}, S^{\prime}=\{a, d\}$, and $R^{\prime}=\emptyset$.

$V(H)$. The complement $\bar{G}$ of a graph $G=(V, E)$ has vertex set $V$ and edge set $\bar{E}=\{x y \mid x y \notin$ $E$ for all distinct $x, y \in V\}$.

A graph $G$ is called $P_{4}$-sparse if every set of five vertices induces at most one $P_{4}$ (chordless path on four vertices) [6]. The efficient recognition of $P_{4}$-sparse graphs is intimately connected to spider graphs, as the next lemma shows.

Lemma 2.1 (7, 8,.). A graph $G$ is $P_{4}$ sparse if and only if exactly one of the following three alternatives is true for every induced subgraph $H$ of $G$ : (i) $H$ is not connected, (ii) $\bar{H}$ is not connected, or (iii) $H$ is a spider

Spiders come in two sub-types, called thin and thick.

Definition 2.2. 8, 11 A graph $G$ is a thin spider if its vertex set can be partitioned into three sets $K, S$, and $R$ so that (i) $K$ is a clique; (ii) $S$ is a stable set; (iii) $|K|=|S| \geq 2$; (iv) every vertex in $R$ is adjacent to all vertices of $K$ and none of the vertices of $S$; and (v) each vertex in $K$ has a unique neighbor in $S$ and vice versa.

A graph $G$ is a thick spider if its complement $\bar{G}$ is thin spider.

The sets $K, S$, and $R$ are usually referred to as the body, the set of legs, and head, resp., of a thin spider, see Fig. 1. The path $P_{4}$ is the only graph that is both a thin and thick spider.

\section{Results}

Spider graphs have characteristic degree sequences. Suppose $G$ is a thin spider. It follows directly from the definition that $\operatorname{deg}(x)=1$ for all $x \in S$ : The leg $x$ is adjacent to a single body vertex and to none of the head vertices (Condition (iv) and (v)). Each body vertex $x \in K \operatorname{has} \operatorname{deg}(x)=|K|+|R|$, since it is adjacent to $1 \mathrm{leg}$, all $|K|-1$ other body vertices, and all $|R|$ head vertices. For a head vertex $x \in R$ we have $|K| \leq \operatorname{deg}(x) \leq|K|+|R|-1$ since it is adjacent at least to all body vertices and at most to all body and head vertices except itself. As $|K|+|R|=|V|-|S|$ we can rephrase these observations in the following form, which have in parts already be discovered in [8, Obs. 2.8 \& 2.9].

Lemma 3.1. If $G$ is a thin spider, then it has $|S| \geq 2$ legs with degree $1,|S|$ body vertices with degree $|V|-|S|$, and $|V|-2|S|$ head vertices $x \in R$ with $2 \leq|S| \leq \operatorname{deg}(x) \leq|V|-|S|-1$.

Our main result is that this condition is also sufficient to identify thin spiders:

Theorem 3.2. A graph $G=(V, E)$ with vertex set $V$ is a thin spider if and only if there is an integer $s \geq 2$ so that $G$ has exactly $s$ vertices of degree 1 and exactly s vertices of degree $|V|-s$.

Proof. The "only if" part of this statement is a consequence of Lemma 3.1

Hence, consider a graph $G$ with $s \geq 2$ nodes with degree 1 and $s$ nodes of degree $|V|-s$. Define $K:=\{x \in V|\operatorname{deg}(x)=| V \mid-s\}$ as the set of body and $S:=\{x \in V \mid \operatorname{deg}(x)=1\}$ as the legs. 
Claim 1. For each body vertex $x \in K$ there is a unique leg $y \in S$ so that $x y \in E$.

If $x$ is not adjacent to a leg $y \in S$ then it has at most $|V|-|S|-1$ neighbors, a contradiction. Thus, every body vertex $x \in K$ has at least one adjacent leg $y \in S$. Since $|K|=|S|$ by assumption, every body node is adjacent to exactly one leg.

$\triangleleft$

This establishes property (v).

Claim 2. Every body vertex $x \in K$ is adjacent to all non-leg vertices $y \in V \backslash S$, except itself.

Since $\operatorname{deg}(x)=|V|-|S|$ and one of the neighbors of $x$ is a leg, it is adjacent to $|V|-|S|-1$ non-leg vertices. There are in total $|V|-|S|$ non-leg vertices, i.e., $|V|-|S|-1$ non-leg vertices other than $x$ itself. Since $x$ cannot be adjacent to itself, it is adjacent to all other non-leg vertices. $\triangleleft$

It follows that $K$ forms a clique in $G$, i.e., property (i) holds. Furthermore every head node $z \in$ $R:=V \backslash(K \cup S)$ is connected to every body node and to none of the legs, i.e., property (iv) holds. Conditions (ii) and (iii) are satisfied by construction, thus $G$ is a thin spider.

Since the complement of a thick spider is a thin spider and vice versa, the characterization of thin spiders immediately implies a characterization of thick spiders because $\operatorname{deg}_{\bar{G}}(x)+\operatorname{deg}_{G}(x)=|V|-1$. It follows that a thick spider has $|S|$ body vertices of degree $|V|-2$ and $|S|$ leg vertices of degree $|S|-1$.

Corollary 3.3. $G$ is a thick spider if and only if there is an integer $s \geq 2$ so that $G$ has exactly $s$ vertices with degree $s-1$ and $s$ vertices with degree $|V|-2$.

Theorem 3.4. Let $\Pi=\left(n_{0}, n_{1}, \ldots, n_{w}\right)$ be an arbitrary degree sequence. Then it holds that $\Pi$ is realizable by a thin spider if and only if there are integers $s \geq 2, v \geq 4, s<v$ so that

(i) $n_{1}=s, n_{v-s}=s$, and

(ii) $\Pi^{\prime}=\left(m_{0}, m_{1}, \ldots, m_{w}\right)$ is a graphical degree sequence where

$$
m_{k}= \begin{cases}n_{k}=0, & \text { if } 2 \leq k<s \text { or } k \geq v-s \\ n_{k}-s, & \text { otherwise. }\end{cases}
$$

A sequence $\left(n_{1}, \ldots, n_{j}, \ldots, n_{|V|-1}\right)$ is the degree sequence of a thick spider if and only if $\left(n_{|V|-1}, \ldots, n_{|V|-1-j}, \ldots, n_{1}\right)$ is the degree sequence of a thin spider.

Proof. Let $\Pi$ be realizable by a thin spider. Theorem 3.2 implies Conditions (i). As already observed, the $|V|-2|S|$ head vertices of a thin spider have degrees in the range from $|S|$ to $|V|-|S|-1$, that is reflected by the given degrees of $\Pi^{\prime}$ in Condition (ii). Moreover, since $\Pi$ is the degree sequence of a thin spider $G, R$ induces a subgraph of $G$ and each vertex in $R$ has $s$ neighbors in $K$, we can conclude that $\Pi^{\prime}$ is a graphical degree sequence. Note, from the algorithmic point of view, Condition (ii) can easily be checked by the Erdős-Gallai Theorem [1].

Now assume that there are integers $s \geq 2, v \geq 4, s<v$ so that Conditions (i) and (ii) are fulfilled for $\Pi$. We construct a spider from the graph $(V, E)$ with $|V|=v$ and $E=\emptyset$. Choose a subset $K \subseteq V$ with $|K|=s, S \subseteq V \backslash K$ with $|S|=s$, and set $R=V \backslash(K \cup S)$. Now, add edge $x y$ to $E$ for all distinct $x, y \in K$, choose a bijection $f: K \rightarrow S$ and add $x f(x)$ to $E$ for all $x \in K$ and finally add edge $a b$ to $E$ for all $a \in K, b \in R$. Clearly, the construction is feasible in terms of Condition (i) and (ii), and fulfills the properties of a thin spider. Finally, Condition (ii) also ensures that $R$ induces itself a graph, that can be constructed with the Havel-Hakimi algorithm [3, 2.

The result for thick spiders follows by observing that the degree sequence of $\bar{G}$ is related to the degree sequence of $G$ via $\bar{n}_{k}=n_{|V|-1-k}$ for $0 \leq k<|V|-1$.

\section{References}

[1] P. Erdős and T. Gallai. Gráfok előírt fokszámú pontokkal. Matematikai Lapok, 11:264-274, 1960.

[2] S.L. Hakimi. On realizability of a set of integers as degrees of the vertices of a linear graph. Journal of the Society for Industrial and Applied Mathematics, 10:496-506, 1962. 
[3] V. Havel. A remark on the existence of finite graphs. C̆asopis pro pěstováni matematiky (in Czech), 80:477-480, 1955.

[4] M. Hellmuth, M. Hernandez-Rosales, K. T. Huber, V. Moulton, P. F. Stadler, and N. Wieseke. Orthology relations, symbolic ultrametrics, and cographs. Journal of Mathematical Biology, 66(1-2):399-420, 2013.

[5] M. Hellmuth, N. Wieseke, M. Lechner, H.-P. Lenhof, M. Middendorf, and P. F. Stadler. Phylogenomics with paralogs. PNAS, 2014. in review.

[6] C. T. Hoàng. Perfect graphs. PhD thesis, School of Computer Science, McGill University, Montreal, Canada, 1985.

[7] B. Jamison and S. Olariu. $p_{4}$-reducible-graphs, a class of uniquely tree representable graphs. Stud. Appl. Math., 81:79-87, 1989.

[8] B Jamison and S Olariu. Recognizing $p_{4}$-sparse graphs in linear time. SIAM J. Comput., 21:381-406, 1992.

[9] Yunlong Liu, Jianxin Wang, Jiong Guo, and Jianer Chen. Cograph editing: Complexity and parametrized algorithms. In B. Fu and D. Z. Du, editors, COCOON 2011, volume 6842 of Lect. Notes Comp. Sci., pages 110-121, Berlin, Heidelberg, 2011. Springer-Verlag.

[10] Yunlong Liu, Jianxin Wang, Jiong Guo, and Jianer Chen. Complexity and parameterized algorithms for cograph editing. Theoretical Computer Science, 461(0):45 - 54, 2012.

[11] James Nastos and Yong Gao. Bounded search tree algorithms for parameterized cograph deletion: Efficient branching rules by exploiting structures of special graph classes. Discr. Math. Algor. Appl., 4:1250008, 2012 .

Sarah Berkemer

Bioinformatics Group,

Department of Computer Science and Interdisciplinary Center for Bioinformatics

University of Leipzig,

Härtelstrasse 16-18, D-04107 Leipzig, Germany

e-mail: bsarah@bioinf.uni-leipzig.de

Ricardo Chaves

Departamento de Ciência da Computação (CIC)

Instituto de Ciências Exatas

Universidade de Brasília

Campus Universitário - Asa Norte

Brasília DF - CEP: 70910-900

Brasil

e-mail: rrcchaves@gmail.com

Adrian Fritz

Center for Bioinformatics

Saarland University

Building E 2.1, Room 413

P.O. Box 151150

D - 66041 Saarbrücken

Germany

e-mail: s9adfrit@stud.uni-saarland.de

Marc Hellmuth

Center for Bioinformatics

Saarland University

Building E 2.1, Room 413

P.O. Box 151150

D - 66041 Saarbrücken

Germany

e-mail: marc@bioinf.uni-leipzig.de 


\author{
Maribel Hernandez-Rosales \\ Departamento de Ciência da Computação (CIC) \\ Instituto de Ciências Exatas \\ Universidade de Brasília \\ Campus Universitário - Asa Norte \\ Brasília DF - CEP: 70910-900 \\ Brasil \\ e-mail: maribel@bioinf .uni-leipzig.de \\ Peter F. Stadler \\ Bioinformatics Group, \\ Department of Computer Science; and Interdisciplinary Center for Bioinformatics, \\ University of Leipzig, \\ Härtelstrasse 16-18, D-04107 Leipzig,Germany \\ Max Planck Institute for Mathematics in the Sciences \\ Inselstrasse 22, D-04103 Leipzig, Germany \\ RNomics Group, Fraunhofer Institut für Zelltherapie und Immunologie, Deutscher Platz 5e, D-04103 Leipzig, \\ Germany \\ Department of Theoretical Chemistry, University of Vienna, Währingerstraße 17, A-1090 Wien, Austria \\ Santa Fe Institute, 1399 Hyde Park Rd., Santa Fe, NM87501, USA \\ e-mail: studla@bioinf .uni-leipzig.de
}

